\title{
Research on low voltage ride through testing method for wind turbine by artificial short circuit
}

\author{
Dong Henan ${ }^{1,2, a^{*}}$, Han Zijiao ${ }^{2, b}$, Yuan shun ${ }^{1, c}$, Sun Feng ${ }^{2, d}$, Zhang Lingjie ${ }^{3, e}$
}

${ }^{1}$ Shenyang University of Technology institute of electrical engineering, Shenyang 110023,China

${ }^{2}$ State Grid Liaoning Electric Power Company Electric Power Research Institute, Shenyang 110006,China

${ }^{3}$ Liaoning electric power communication engineering company, Shenyang 110023,China

a13555878500@163.com, bthuwhatever@163.com, yuanshun@serc.gov.cn, ddarkmars_2000@126.com, ${ }^{\mathrm{e}} \mathrm{zhanglj@163.com}$

Keywords: Low voltage ride through, artificial short circuit, wind turbine, wind farm

Abstract. In recent years, the wind power industry in China developed rapidly, by the people's attention. The development of wind power has brought us considerable economic benefits and significant social benefits, but also to the stability of power system has brought a significant impact. Low voltage ride through capability of wind turbine is one of the important factors that affect the safety of grid. China's wind farms are widely distributed. Low voltage ride through test method of conventional wind turbine has a long time and a slow progress. In this paper, a new method is proposed to realize the low voltage ride through performance test of wind turbine, and the validity and accuracy of the testing method are verified by the simulation results.

\section{Introduction}

China's new energy power generation proportion is growing, especially wind power generation. People pay more and more attention to the low voltage ride through testing technology of wind turbine[1]. The conventional low voltage ride through testing technology of wind turbine is mainly based on the test equipment, including the test system developed by w2ps and the test system of German FGH. The method of low voltage ride through the test system has the disadvantages of high cost, long time and so on. In this paper, a new method is proposed to realize the low voltage ride through performance test of wind turbine, and the validity and accuracy of the testing method are verified by the simulation results[2].

\section{Principle of artificial short circuit test}

We can use the short circuit test method to verify the low voltage ride through of wind farm. This principle is based on the natural conditions of wind farm. On the $35 \mathrm{kV}$ set of the wind farm, the artificial short circuit test is carried out, and the $35 \mathrm{kV}$ bus voltage drop depth is controlled by the distance of the short circuit point range[3-4]. Test to assess the ability of low voltage ride through the wind turbine. The basic principle of short circuit test is shown in figure 1.

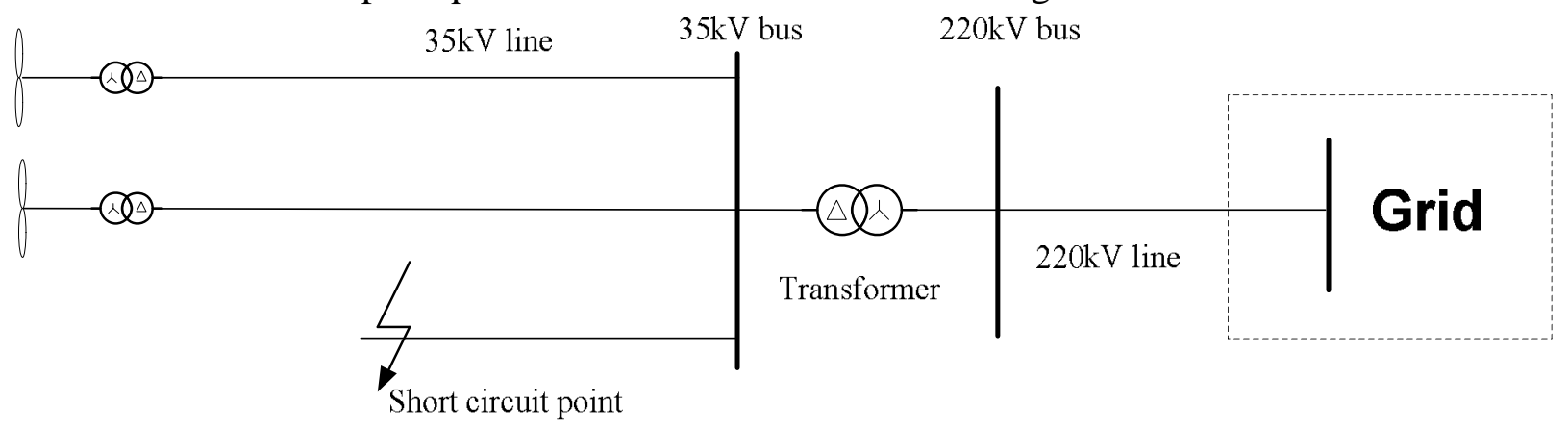

Fig.1The basic schematic of the short-circuit test 
Compared with the use of test equipment for testing the way, artificial short circuit method has many advantages. This test method is low cost and short period, while capable of detecting low-voltage ride-through capability of the entire wind farm. Not only to verify the fan, but also verify LVRT capability of some other devices. But there are also some disadvantages, it can not precisely control the voltage drop time, but it also restricted the grid structure and there are some security risks.

\section{Artificial short circuit test model}

Asymmetrical power system failure, the elements bear through the three-phase voltage and phase current will be asymmetric. It is assumed that the parameters of the power system are linear, with the symmetrical component method, and the asymmetric voltage and current are decomposed into three groups of symmetrical components. Three-phase fundamental phasor $\mathrm{F}$ (may represent a U or I), which can be used symmetrical fundamental component positive, negative and zero sequence. Formula is as follows.

$$
\begin{aligned}
& \left\{\begin{array}{l}
\dot{F}_{a}=\dot{F}_{a(1)}+\dot{F}_{a(2)}+\dot{F}_{a(0)} \\
\dot{F}_{b}=\dot{F}_{b(1)}+\dot{F}_{b(2)}+\dot{F}_{b(0)} \\
\dot{F}_{c}=\dot{F}_{c(1)}+\dot{F}_{c(2)}+\dot{F}_{c(0)}
\end{array}\right. \\
& \left\{\begin{array}{l}
\dot{F}_{a}=\dot{F}_{a(1)}+\dot{F}_{a(2)}+\dot{F}_{a(0)} \\
\dot{F}_{b}=a^{2} \dot{F}_{a(1)}+a \dot{F}_{a(2)}+\dot{F}_{a(0)} \\
\dot{F}_{c}=a \dot{F}_{a(1)}+a^{2} \dot{F}_{a(2)}+\dot{F}_{a(0)}
\end{array}\right.
\end{aligned}
$$

Among them, $\dot{F}_{a(1)}, \dot{F}_{b(1)}$ and $\dot{F}_{c(1)}$ show the positive sequence component, $\dot{F}_{a(2)}, \dot{F}_{b(2)}$ and $\dot{F}_{c(2)}$ indicates the negative sequence components; $\dot{F}_{a(0)}, \dot{F}_{b(0)}$ and $\dot{F}_{c(0)}$ represents zero sequence components. Positive sequence components and negative sequence components are symmetrical. Zero-sequence each component equal and the same direction.
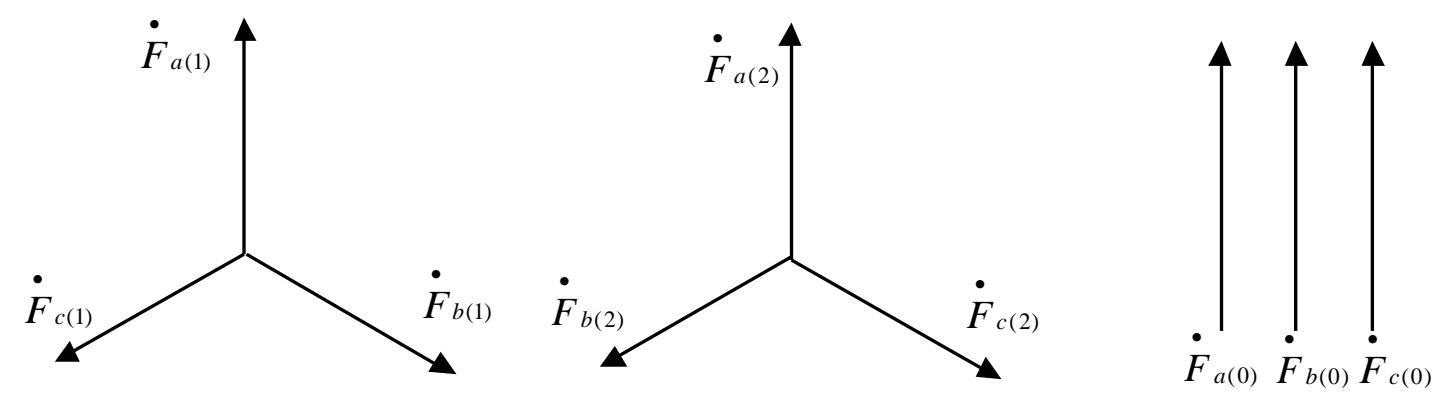

Fig.2 Symmetrical components

The above formula can be changed:

$\left\{\begin{array}{l}\dot{F}_{a}=\dot{F}_{a(1)}+\dot{F}_{a(2)}+\dot{F}_{a(0)} \\ \dot{F}_{b}=a^{2} \dot{F}_{a(1)}+a \dot{F}_{a(2)}+\dot{F}_{a(0)} \\ \dot{F}_{c}=a \dot{F}_{a(1)}+a^{2} \dot{F}_{a(2)}+\dot{F}_{a(0)}\end{array}\right.$

Among them, $\alpha=e^{j 120^{\circ}}=-\frac{1}{2}+j \frac{\sqrt{3}}{2}, \alpha^{2}=e^{j 240^{\circ}}=-\frac{1}{2}-j \frac{\sqrt{3}}{2}$. Represented as a matrix form 


$$
\left[\begin{array}{c}
\dot{F_{a}} \\
\dot{F_{b}} \\
\dot{F_{c}}
\end{array}\right]=\left[\begin{array}{ccc}
1 & 1 & 1 \\
a^{2} & a & 1 \\
a & a^{2} & 1
\end{array}\right]\left[\begin{array}{c}
\dot{F_{a(1)}} \\
\dot{F_{b(2)}} \\
\dot{F_{c(0)}}
\end{array}\right]
$$

In the three-phase symmetrical impedance circuit, three symmetrical component system is independent of each other, so it can be independent of positive sequence, negative sequence and zero sequence network. Short circuit calculation, zero-sequence equivalent circuit model should pay attention to the problem of zero sequence current path[5-7]. The zero sequence current is related to the three-phase connection mode of the element and the grounding mode of the neutral point. This point should pay special attention to the zero sequence equivalent circuit of the transformer.Zero-sequence equivalent circuit of the power system should include all elements of the zero-sequence current under fault conditions can be adopted, but not including zero-sequence current can not flow elements.

Any type of short circuit of positive, negative and zero sequence fault component network shown in Figure 3, in which Fig. (a) may be made (b), (c), (d) and (e) superposition.

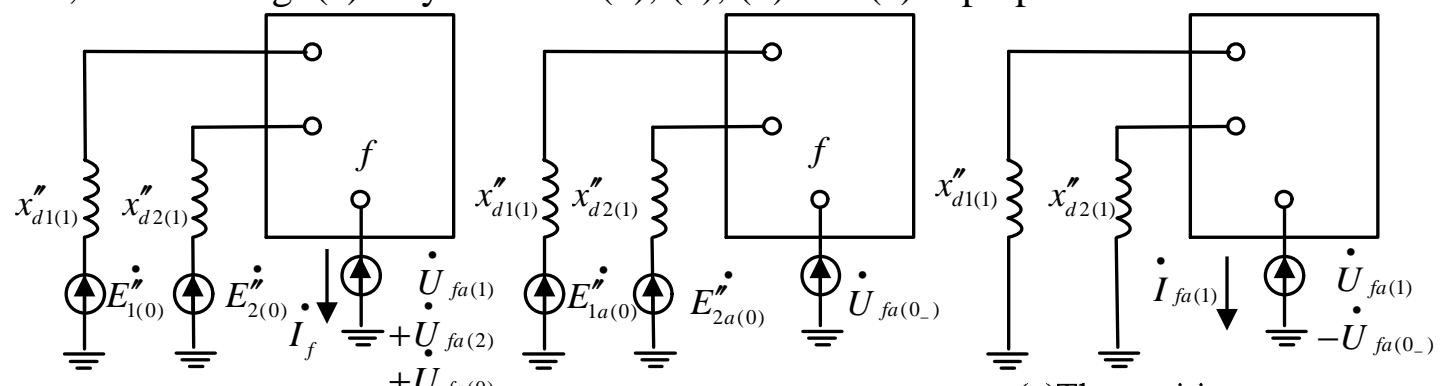

(a)A phase schematic after failure

(b)Normal positive sequence

(c)The positive sequence

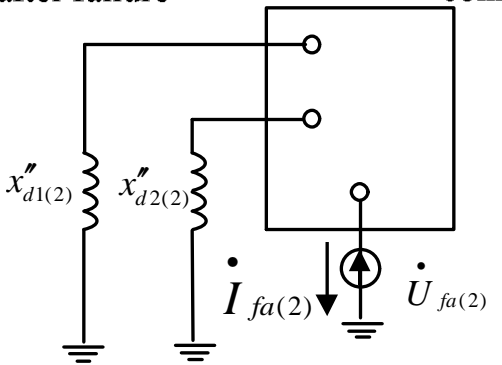

(d)Negative sequence fault component network

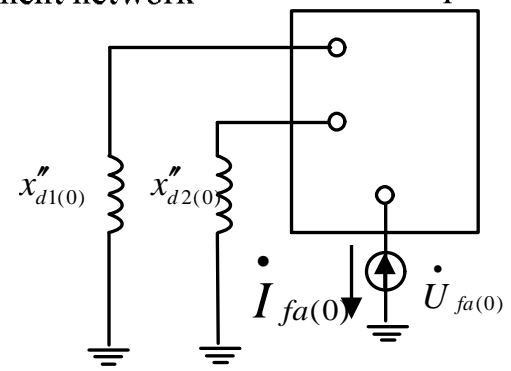

(e)Zero sequence fault component network
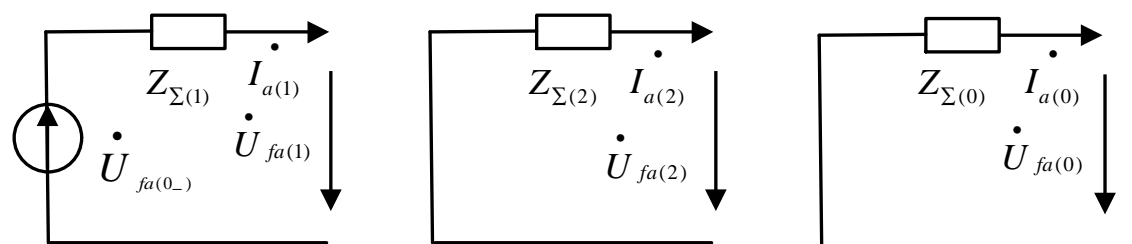

(f)Each sequence equivalent circuit troubleshooting

Fig.3 Fault network and sequence fault component network

In Fig. (f) as shown in the equivalent circuit, A phase short circuit voltage of each sequence is

$\left\{\begin{array}{l}\dot{U}_{f a(1)}=\dot{U}_{f a\left(0_{-}\right)}-Z_{\Sigma(1)} \dot{I}_{f a(1)} \\ \dot{U}_{f a(2)}=-Z_{\Sigma(2)} \dot{I}_{f a(2)} \\ \dot{U}_{f a(0)}=-Z_{\Sigma(0)} \dot{I}_{f a(0)}\end{array}\right.$ 
Formula $5, Z_{\Sigma(1)}=Z_{f f(1)} 、 Z_{\Sigma(2)}=Z_{f f(2)}$ and $Z_{\Sigma(0)}=Z_{f f(0)}$, respectively, equivalent positive sequence impedance of the whole network to short-circuit point, the negative sequence impedance and zero sequence impedance, $\dot{U}_{f a\left(0_{-}\right)}$is short circuit voltage value before the failure occurred[8].

Combined with the artificial short circuit experiment, the calculation method of the two phase ground fault is analyzed. For example, the BC phase to ground fault, as shown in figure 4.

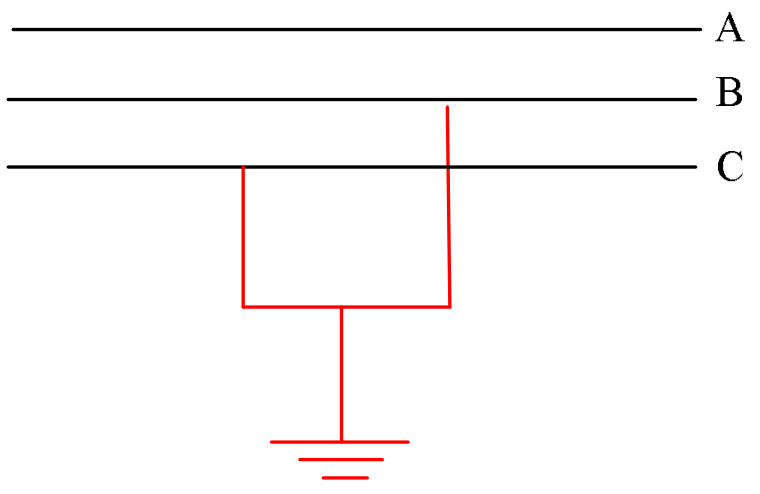

Fig.4 The fault graph of B 、 C phase to ground

Phasor represents B, C-phase short boundary conditions:

$$
\left\{\begin{array}{l}
\dot{I}_{f a}=0 \\
\dot{U}_{f b}=\dot{U}_{f c}=0
\end{array}\right.
$$

Boundary Conditions is represented by sequence component

$$
\left\{\begin{array}{l}
\dot{I}_{f a(1)}+\dot{I}_{f a(2)}+\dot{I}_{f a(0)}=0 \\
\dot{U}_{f a(1)}=\dot{U}_{f a(2)}=\dot{U}_{f a(0)}
\end{array}\right.
$$

Its complex sequence network diagram shown in Figure 5:

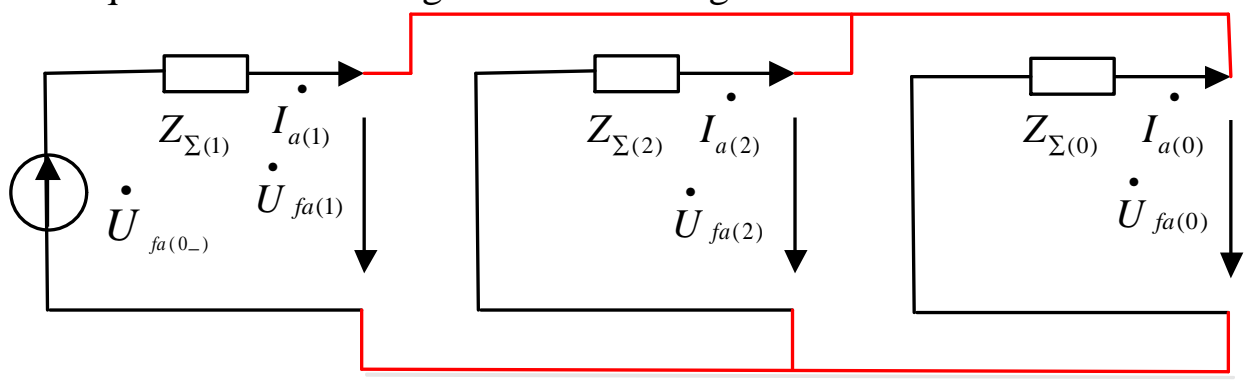

Fig.5 The diagram of composite sequence network in two-phase(B、C) ground fault directly Sequence current of fault:

$$
\left\{\begin{array}{l}
\dot{I}_{f a(1)}=\frac{\dot{U}_{f a\left(0_{-}\right)}}{Z_{\Sigma(1)}+\frac{Z_{\Sigma(2)} Z_{\Sigma(0)}}{Z_{\Sigma(2)}+Z_{\Sigma(0)}}} \\
\dot{I}_{f a(2)}=-\dot{I}_{f a(1)} \frac{Z_{\Sigma(0)}}{Z_{\sum(2)}+Z_{\Sigma(0)}} \\
\dot{I}_{f a(0)}=-\dot{I}_{f a(1)} \frac{Z_{\Sigma(2)}}{Z_{\Sigma(2)}+Z_{\Sigma(0)}}
\end{array}\right.
$$

Troubleshooting sequence voltage calculated according to the formula (5) 


$$
\dot{U}_{f a(1)}=\dot{U}_{f a(2)}=\dot{U}_{f a(0)}=\dot{I}_{f a(1)} \frac{Z_{\Sigma(2)} Z_{\Sigma(0)}}{Z_{\Sigma(2)}+Z_{\Sigma(0)}}=\dot{U}_{f a(0-)} \frac{Z_{\Sigma(2)} Z_{\Sigma(0)}}{Z_{\Sigma(1)} Z_{\Sigma(2)}+Z_{\Sigma(1)} Z_{\Sigma(0)}+Z_{\Sigma(2)} Z_{\Sigma(0)}}
$$

Short-circuit test calculation, line and transformer impedance model error is smaller.

\section{Artificial short-circuit test experiment and simulation}

Short-circuit test conducted in May 2012. The wind farm is located in the Inner Mongolia Autonomous Region, and sent to the A substation by $220 \mathrm{kV}$ line. The capacity of the wind farm is 49.5MW, which is composed of $331.5 \mathrm{MW}$ doubly fed wind power generation units. Three line access to the main transformer low voltage side, where the main transformer capacity 120MVA.

Short circuit point selection as fig. 6 .

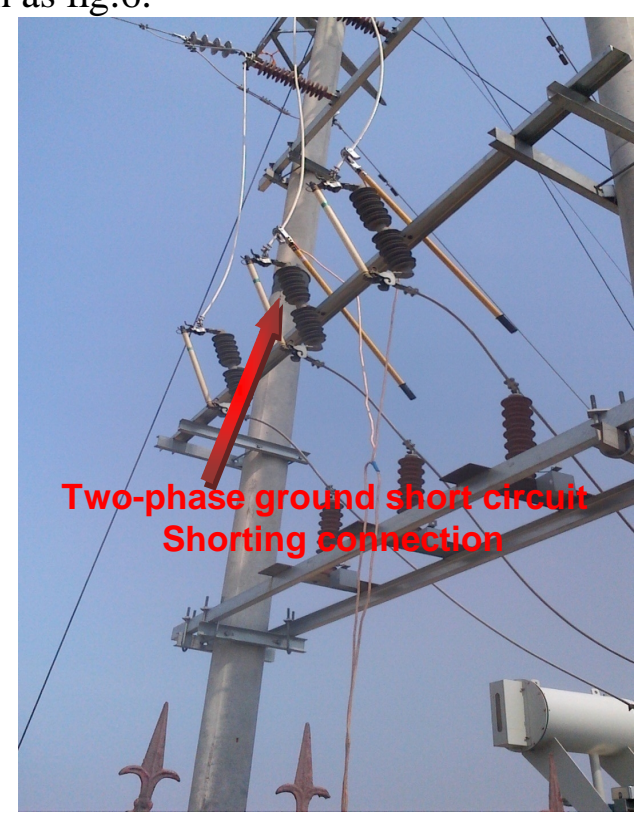

Fig.6 The actual choice of short circuit point

Short Circuit Duration 600ms, and the voltage and current of the normal set is shown in the following diagram. Before the experiment, A phase voltage is $1.017 \mathrm{pu}, \mathrm{B}$ phase voltage is $1.019 \mathrm{pu}, \mathrm{C}$ phase voltage is $1.014 \mathrm{pu}, \mathrm{A}$-phase current is $61 \mathrm{~A}, \mathrm{~B}$ phase voltage is $60 \mathrm{~A}$, C-phase current is $63 \mathrm{~A}$; In the experiment, the phase voltage of A was 1.198 times before the experiment, the $\mathrm{B}$ phase voltage was 0.295 times before the experiment, the $\mathrm{C}$ phase voltage was 0.297 times before the experiment, the current A phase was 0.885 times before the experiment, the B phase voltage was 3.400 times before the experiment, and the $\mathrm{C}$ phase current was 3.111 times before the experiment.

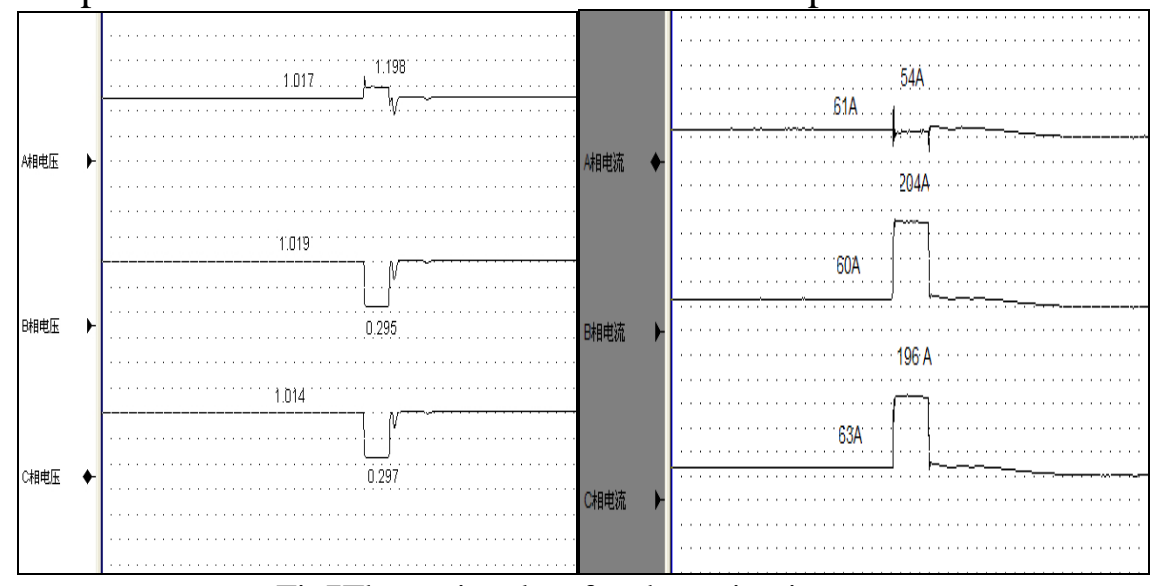

Fig7The testing data for short-circuit 
Experimental results: the running of the wind turbine does not run off the network, to meet the requirements of low voltage ride through. In DIgSILENT / Power Factory in modeling, simulation results are shown below.

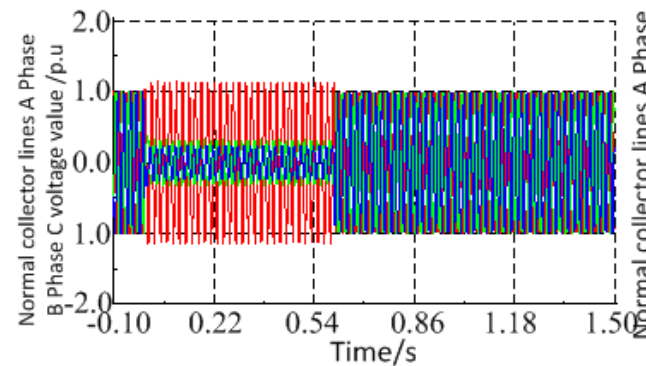

a)The value of voltage of the normal collecting power lines A B C phase

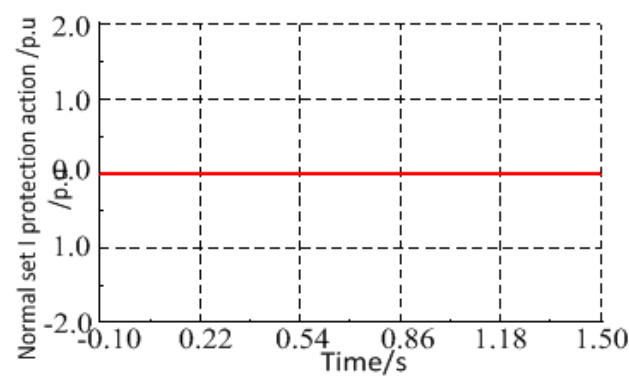

c) The normal collector I line protection value

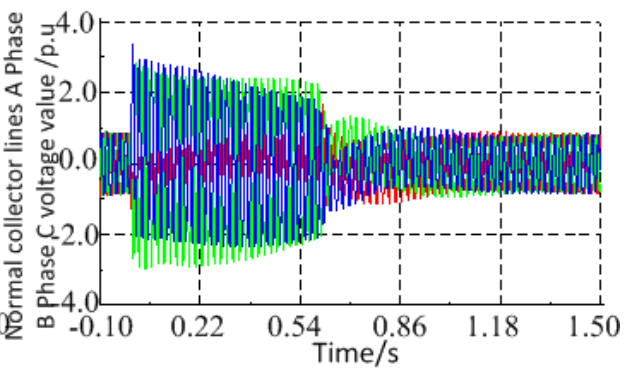

b) The current of voltage of the normal collecting power lines A B C phase

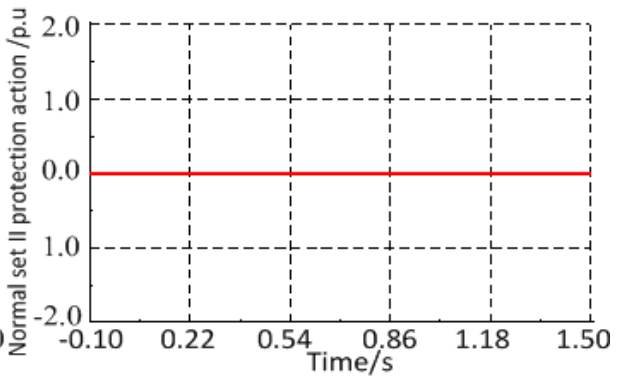

d) The normal collector II line protection value

Fig.8The simulation results of short-circuit test

As can be seen from the picture, the wind turbine has not removed. The simulation results are in agreement with the measured results.

\section{Conclusion}

A new method is proposed to realize the low voltage ride through performance test of wind turbine. Combining test wind farm in Inner Mongolia, as well as analysis of simulation results to verify the validity and accuracy of the test method. To provide a new way to carry out the work.

\section{References}

[1] S. M. Muyeen, Junji Tamura, Toshiaki Murata. Stability Augmentation of a Grid-connected Wind Farm. London: Springer, 2008.

[2] Verband der netzbetreiber. Network and System Rules of the German Transmission System Operators, 2003.

[3] Xing Zuoxia, Dong Henan, Cheng Xuke,et al. Research the Example of Reactive Power Based on DIgSILENT When Wind Farm Integrated Into the Power System. Electrical Engineering , 2012 , ( 8 ) 10-13.

[4] Dong Henan. research Of LVRT characteristics of Wind turbine and dynamic reactive power compensation technology . Shenyang University of Technology , 2013 .

[5] Akhmatov,V. ,Knudsen,H. ,Bruntt , M. ,Nielsen,A.H.(2000(b)).Advanced simulation of windmills in the electric power supply, Electrical Power and Energy Systems , vol.22.no.6 , pp421-434. 
[6] Seman S,Niiranen J,Arkkio A. Ride-throngh analysis of doubly fed induction wind-power generator under unsymmetrical network disturbance. IEEE Transactions on Power Systems , 2006 , 21(4):1782-1789.

[7] Xu L,Wang Y. Dyanmic modeling and control of DFIG-based wind turbines under unbalanced network conditions. IEEE Transactions on Power Systems , 2007 , 22(1):314-323.

[8] Lopez J,Gubia E,Sanchis P,et al. Wind turbines based doubly fed induction generator under asymmetrical voltage dips. IEEE Transactions on Energy Conversion , 2008 , 23(1):321-330. 